Revista Destaques Acadêmicos, Lajeado, v. 11, n. 3, 2019. ISSN 2176-3070

DOI: http://dx.doi.org/10.22410/issn.2176-3070.v11i3a2019.2171

http://www.univates.br/revistas

\title{
DETECÇÃO DE PESTICIDAS ORGANOCLORADOS NA ÁGUA E A ASSOCIAÇÃO DA EXPOSIÇÃO HUMANA À ESSES POLUENTES COM O RISCO DE DIABETES MELLITUS TIPO 2
}

\author{
Ana Paula Guimarães da Costa Carvalho ${ }^{1}$, Diego Pereira ${ }^{1}$, \\ Edvane de Alcantara Borges ${ }^{1}$, Giovane Pereira de Oliveira ${ }^{1}$, \\ Maria Caroline Nazaré Pinto dos Santos ${ }^{1}$, Roberta Oliveira de Araújo Morais ${ }^{1}$, \\ Andreza Lopes Maia², Diene Conceição Poiares Aranha ${ }^{3}$, \\ Laine Celestino Pinto ${ }^{4}$, Sylvia de Fátima dos Santos Guerra ${ }^{5}$
}

Resumo: Os pesticidas organoclorados (PCOs) são poluentes orgânicos persistentes (POPs) que constituem uma grande fonte de contaminação da água, solo e ar. A exposição humana a eles pode ser um fator de risco para a diabetes mellitus tipo 2 (DM2), sendo essa associação atribuída a mecanismos de ação dos PCOs que possivelmente influenciam na homeostase da glicose. O objetivo do presente estudo é realizar uma revisão sistemática descritiva da literatura sobre a detecção de PCOs na água e a associação da exposição humana à esses poluentes com o risco de DM2. A busca de artigos foi realizada de março a abril de 2018 nas bases de dados PubMed, Bireme e SciELO, utilizando os descritores "organochlorines and water" e "organochlorines and diabetes". Foram obtidos 477 artigos, sendo 455 (PubMed), 18 (Bireme) e 4 (SciELO), onde 15 foram incluídos na revisão e 462 foram excluídos. Os estudos de detecção de PCOs na água relataram os diclorodifeniltricloroetanos (DDTs), hexaclorohexanos (HCHs), heptacloros

1 Acadêmicos do curso de Biomedicina, Centro Universitário Metropolitano da Amazônia, Belém, PA.

2 Biomédica, Mestre em Análises Clínicas Profissional (UFPA), Docente do curso de Biomedicina, Centro Universitário Metropolitano da Amazônia, Belém, PA.

3 Tecnóloga em Radiologia, Mestre em Virologia (IEC/SVS/MS), Docente do curso de Biomedicina, Centro Universitário Metropolitano da Amazônia, Belém, PA.

4 Biomédica, Doutora em Genética e Biologia Molecular (UFPA), Docente do curso de Biomedicina, Centro Universitário Metropolitano da Amazônia, Belém, PA.

5 Farmacêutica-Bioquímica, Doutora em Doenças Tropicais (NMT/UFPA), Docente do curso de Biomedicina, Centro Universitário Metropolitano da Amazônia, Belém, PA. 
como os mais associados aos riscos ecotoxicológicos e à saúde humana quando em concentrações acima dos padrões estabelecidos. Em relação aos estudos de associação de PCOs com DM2, os DDTs, HCHs e HCB foram os mais associados positivamente ao risco de DM2. Esses achados alertam sobre a responsabilidade social acerca do meio ambiente, enfatizando a criação de estudos e programas de monitoramento ambiental para controle do uso desses PCOs, bem como a criação de estudos futuros que busquem entender a fisiopatologia da DM2 associada aos PCOs, já que essa causalidade ainda não foi bem estabelecida.

Palavras-chave: Pesticidas Organoclorados. Contaminação da Água. Diabetes Mellitus Tipo 2.

\section{INTRODUÇÃO}

Os pesticidas organoclorados (PCOs) são um grupo de poluentes orgânicos persistentes (POPs) que representam uma ameaça aos ecossistemas e à saúde humana, devido as suas características de alta persistência no meio ambiente, bioacumulação em tecidos orgânicos, biomagnificação, meia vida longa e toxicidade crônica, que aumentam a sua capacidade de contaminar e causar efeitos adversos em humanos, animais e vegetais (JONES; VOOGT, 1999 citado por HUANG et al., 2013; ZHOU et al., 2006).

Esses compostos sintéticos de cadeia cíclica caracterizam-se por apresentar em sua estrutura química um ou mais átomos de cloro ligados ao carbono, e dentre os principais PCOs conhecidos, podem ser destacados os DDT (diclorodifeniltricloroetano), DDD (diclorodifenildicloroetano), HCB (Hexaclorobenzeno), HCH (Hexaclorociclohexano), Oxyclordano, transNonacloro, Endosulfan, Heptacloro, Aldrin e Dieldrin (DEL GRANDE; REZENDE; ROCHA, 2003; PRATES; GEBARA; POPPI, 2011; JAYARAJ; MEGHA; SREEDEV, 2016).

O uso de PCOs foi proibido em diversos países há muitos anos, onde eles eram utilizados em diversas atividades, principalmente na agricultura, para repelir pragas domésticas e também na saúde pública, para o combate de vetores de doenças infecciosas. No entanto, por possuírem difícil degradação por processos naturais, tornaram-se ao longo dos anos, uma grande fonte de contaminação da água, solo e ar (BAHIA; GUIMARÃES; ASMUS, 2014; JAYARAJ; MEGHA; SREEDEV, 2016; MOSTAFOLOU; ABDOLLAHI, 2016).

A exposição humana à esses PCOs pode ocorrer de diversas maneiras, visto que esses compostos são amplamente encontrados no meio ambiente, e chegam à ele através de vários processos como: as atividades agroindustriais em águas costeiras; descarga direta de águas residuais urbanas em rios; efluentes de esgotos; lixiviação pelas chuvas; distribuição pelo vento e incorporação na superfície terrestre por meio do tratamento de sementes, pulverização das folhas, caules de vegetais e frutos (FLORES et al., 2004; RISSATO et al., 2004; HUANG et al., 2013; VARGAS-GONZÁLEZ et al., 2016). 
Apesar de alguns benefícios que trazem os PCOs, intoxicações por esses compostos gera preocupações, pois podem desencadear uma série de efeitos cumulativos e prejudiciais a curto e longo prazo, como o risco de ocorrência de câncer, danos aos sistemas nervoso, cardíaco e endócrino, lesões hepáticas e renais e discrasias sanguíneas (DEL GRANDE; REZENDE; ROCHA, 2003; FLORES et al., 2004; RISSATO et al., 2004; PRATES; GEBARA; POPPI, 2011; BAHIA; GUIMARÃES; ASMUS, 2014).

Alguns estudos também associam a contaminação por PCOs com o desenvolvimento de síndromes metabólicas, principalmente a diabetes mellitus tipo 2 (DM2), que é responsável por 90 a 95\% dos casos de diabetes e está diretamente relacionada a vários fatores de risco. Essa associação pode ser atribuída a diversos mecanismos de ação dos PCOs, que interferem nas funções pancreáticas afetando a secreção de insulina e influenciando na homeostase da glicose, além de outros mecanismos que reduzem os efeitos metabólicos, mas que ainda são alvo de estudos (TANG et al., 2014; JUNTARAWIJIT; JUNTARAWIJIT, 2018).

Portanto, devido a sua característica de persistência no meio ambiente e sua tendência bioacumuladora em humanos, torna-se necessário o desenvolvimento de estudos que façam o monitoramento dos PCOs, principalmente na água, que é um elemento fundamental para a manutenção da vida e constitui um importante reservatório de contaminação, e em humanos, para associar a exposição aos PCOs com o desenvolvimento de diversas patologias, buscando assim proteger o meio ambiente e a saúde humana.

Acerca disso, o objetivo do presente estudo é realizar uma revisão sistemática descritiva da literatura sobre a detecção de pesticidas organoclorados na água e a associação da exposição humana à esses poluentes com o risco de diabetes mellitus tipo 2 .

\section{MATERIAL E MÉTODOS}

A busca de artigos para o presente estudo foi realizada no período de março a abril de 2018, onde foram analisadas as bases de dados National Library of Medicine (PubMed), Biblioteca Virtual em Saúde (Bireme/BVS) e Scientific Electronic Library Online (SciELO). Os descritores utilizados foram: "organochlorines and water" e "organochlorines and diabetes". Para a seleção dos artigos, foram estabelecidos os seguintes critérios de inclusão: artigos nos idiomas inglês e português; artigos publicados no período de 2013 a 2018; e artigos de estudos realizados apenas com humanos. Os critérios de exclusão foram: artigos que não abordassem a temática proposta no estudo; artigos de revisão; relatos de caso; artigos em duplicata; artigos que abordassem a deteç̧ão simultânea de pesticidas organoclorados e outras substâncias na água; e trabalhos que detectaram pesticidas organoclorados em humanos e associaram com outros tipos de diabetes mellitus além da DM2. 


\section{RESULTADOS}

A busca nas bases de dados para o descritor "organochlorines and water" resultou em 350 artigos e, para o descritor "organochlorines and diabetes", foram encontrados 127 artigos, totalizando assim 477 artigos encontrados nas três bases de dados analisadas. Dos 477 artigos, 95,39\% (455/477) foram originados do PubMed, 3,77\% (18/477) do Bireme e 0,84\% (4/477) do SciELO. Foram selecionados para a presente revisão 15 artigos, e foram excluídos 462 , pois não preencheram os critérios de inclusão. O esquema do processo de busca e seleção dos artigos para o presente estudo é mostrado no fluxograma 1 a seguir:

Fluxograma 1: Esquema do processo de busca e seleção dos artigos

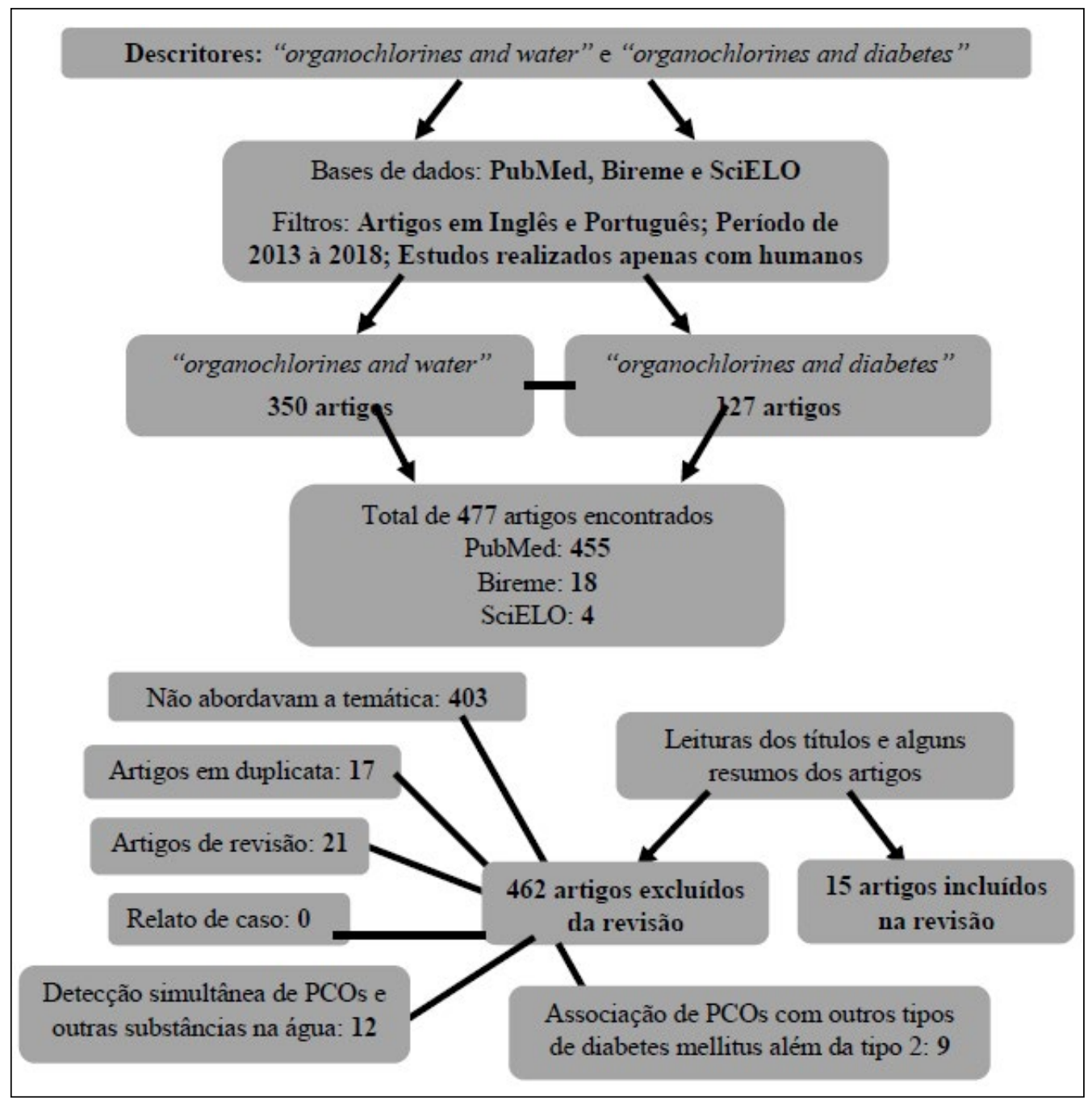


Dos 15 artigos incluídos nesse estudo, 100\% (15/15) foram originados da base de dados PubMed. Em relação aos anos de publicação e idioma, foram incluídos artigos no período de 2013 a 2017, todos em língua inglesa. Os 6 artigos obtidos no ano de 2018 foram excluídos do estudo pois não abordavam a temática proposta. A quantidade e percentual de artigos incluídos por ano de publicação são mostrados no Gráfico 1 a seguir:

Gráfico 1: Quantidade de artigos incluídos no estudo por ano de publicação

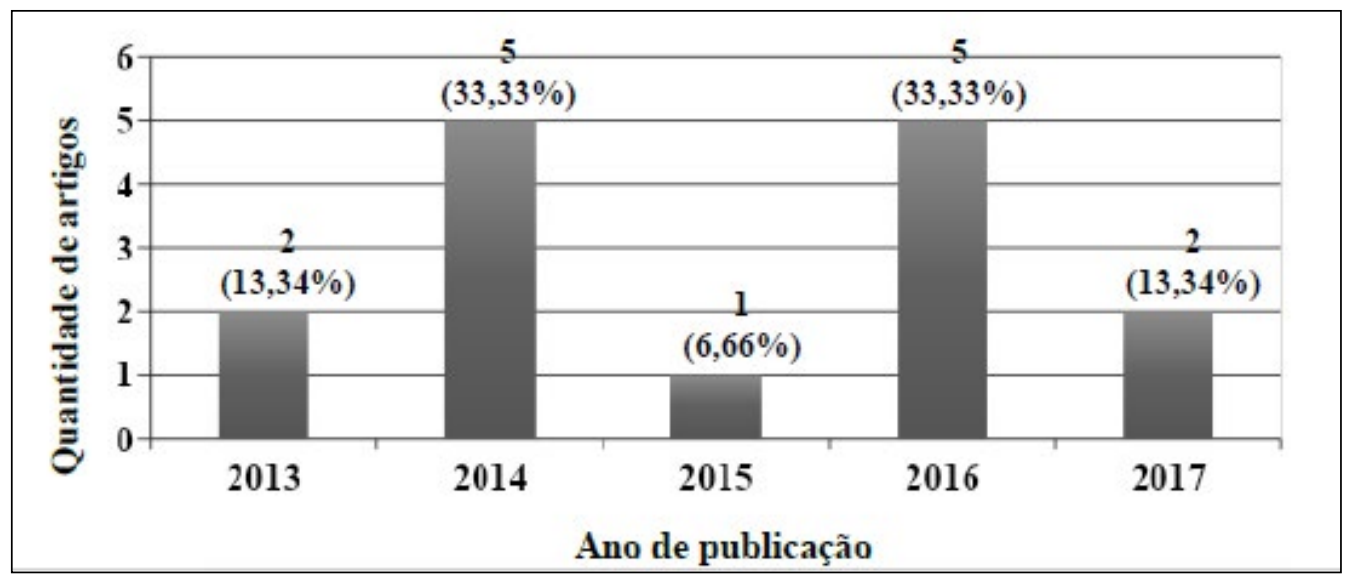

Em relação aos artigos que abordam sobre a detecção de PCOs na água, foram incluídos no presente estudo $46,64 \%$ dos artigos (7/15). Desses 7 artigos, $5(71,42 \%)$ foram estudos realizados na China, $1(14,29 \%)$ foi realizado na Nigéria e $1(14,29 \%)$ na Etiópia, e dentre os PCOs detectados nos estudos, os mais encontrados foram os DDTs, HCHs, HCB, Heptacloros, Aldrinas e Clordanos. A descrição dos 7 artigos é detalhada no Quadro 1 abaixo:

Quadro 1: Descrição dos 7 artigos que abordam sobre a detecção de PCOs na água

\begin{tabular}{|l|c|c|c|}
\hline \multicolumn{1}{|c|}{ Autor/ano } & $\begin{array}{c}\text { Local do } \\
\text { estudo }\end{array}$ & $\begin{array}{c}\mathbf{N}^{\mathbf{o}} \text { de } \\
\text { amostras }\end{array}$ & PCOs mais detectados \\
\hline CUI; WEI; WANG, 2016 & China & 112 & DDTs e HCHs \\
\hline GAO et al., 2015 & China & 26 & HCB, DDTs e HCHs \\
\hline LIU et al., 2016 & China & 28 & HCHs e DDTs \\
\hline $\begin{array}{l}\text { OGBEIDE; TONGO; } \\
\text { EZEMONYE, 2016 }\end{array}$ & Nigéria & 216 & HCHs e DDTs \\
\hline $\begin{array}{l}\text { TEKLU; ADRIAANSE; } \\
\text { BRINK, 2016 }\end{array}$ & Etiópia & 15 & heptacloros, clordanos e HCBs \\
\hline WU et al., 2014 & China & 27 & HCHs, DDTs e aldrinas \\
\hline YANG et al., 2013 & China & 108 & HCHs, DDTs e heptacloros \\
\hline
\end{tabular}


No que diz respeito aos artigos que fizeram a detecção de pesticidas organoclorados em humanos e associaram com o risco de diabetes mellitus tipo 2, foram incluídos 53,36\% dos artigos (8/15), sendo que desses 8, $3(37,5 \%)$ foram estudos desenvolvidos nos Estados Unidos (EUA), 2 (25\%) foram realizados na Arábia Saudita, 2 (25\%) na Coréia e 1 (12,5\%) na Espanha. Dentre os PCOs encontrados nos estudos, os mais detectados foram os HCHs, DDTs, $\mathrm{HCB}$, trans-Nonacloro e Clordanos, e a média do número de participantes dos estudos foi de 295. O Quadro 2 abaixo mostra detalhadamente a descrição dos 8 artigos:

Quadro 2: Descrição dos 8 artigos que detectaram PCOs em humanos e associaram com o risco de diabetes mellitus tipo 2

\begin{tabular}{|l|c|c|c|}
\hline \multicolumn{1}{|c|}{ Autor/ano } & Local do estudo & $\begin{array}{c}\mathbf{N}^{\circ} \text { de } \\
\text { pesquisados }\end{array}$ & PCOs mais detectados \\
\hline $\begin{array}{l}\text { AL-OTHMAN; } \\
\text { ABD-ALRAHMAN; } \\
\text { AL-DAGHRI, 2014 }\end{array}$ & Arábia Saudita & 280 & HCHs \\
\hline AL-OTHMAN et al., 2014 & Arábia Saudita & 280 & DDTs \\
\hline AMINOV et al., 2016 & EUA & 601 & DDE, HCB e Mirex \\
\hline ARREBOLA et al., 2013 & Espanha & 386 & DDTs e HCHs \\
\hline EDEN et al., 2014 & EUA & 263 & $\begin{array}{c}\text { DDE, trans-nonacloro } \\
\text { e oxyclordano }\end{array}$ \\
\hline GRICE et al., 2017 & EUA & 300 & $\begin{array}{c}\text { DDTs, trans-nonacloro } \\
\text { e oxyclordano }\end{array}$ \\
\hline KIM et al., 2014 & Coréia & 50 & DDTs, HCHs e HCB \\
\hline LEE et al., 2017 & Coréia & 200 & DDTs \\
\hline
\end{tabular}

\section{DISCUSSÃO}

A detecção e o monitoramento de PCOs e seus resíduos na água constitui um importante fator de avaliação de risco ecotoxicológico para a saúde humana. Como a água é um importante e indispensável recurso natural, constitui um dos principais meios de exposição a esses poluentes, que, quando encontrados em concentrações elevadas na água, podem contaminá-la, afetando diretamente as formas de vida aquáticas, tornando-a imprópria para o consumo humano e para outras atividades.

Em um estudo realizado com águas de abastecimento e de um lago na cidade de Wuhan, China, Cui; Wei; Wang, (2016) detectaram a presença de PCOs, demonstrando que as concentrações médias mais elevadas nos dois tipos de água analisados foram de DDTs $\left(2,57 \mathrm{ng} / \mathrm{L}^{-1}\right)$ e HCHs $\left(2,31 \mathrm{ng} / \mathrm{L}^{-1}\right)$. Tais concentrações foram relativamente baixas em relação aos padrões de qualidade de águas superficiais exigidos, que determinavam que níveis como 
os de DDTs, heptacloro e aldrina devem ser inferiores a 1.000 , 520 e $3.000 \mathrm{ng} / \mathrm{L}^{-1}$ respectivamente, concluindo que as concentrações encontradas no estudo não ofereciam risco de toxicidade para a vida aquática.

Os achados de Cui; Wei; Wang, (2016) foram semelhantes aos de outro estudo realizado na China por Liu e colaboradores., (2016), que examinaram a distribuição de PCOs ao longo do rio Hanjiang. Os resultados demonstraram níveis de HCHs que variaram de 4,34 a $28,38 \mathrm{ng} / \mathrm{L}^{-1}$ seguido dos DDTs que foram de 6,62 a $35,62 \mathrm{ng} / \mathrm{L}^{-1}$, concluindo que esses níveis também não previam risco ecotoxicológico para a saúde humana, já que estavam em conformidade com os valores padrões estabelecidos, que são de $\leq 2.000 \mathrm{ng} / \mathrm{L}^{-1}$ para os $\mathrm{HCHs}$ $\mathrm{e} \leq 1.000 \mathrm{ng} / \mathrm{L}^{-1}$ para os DDTs.

Gao e colaboradores., (2014) avaliaram a contaminação do rio Liao na China, a partir da detecção de PCOs em sedimentos obtidos das amostras de água. As maiores concentrações médias encontradas foram de $\operatorname{HCB}(4,38 \mathrm{ng} / \mathrm{g}-1)$, $\mathrm{HCHs}(4,01 \mathrm{ng} / \mathrm{g}-1)$ e DDTs $(3,07 \mathrm{ng} / \mathrm{g}-1)$, sendo os dois primeiros os mais prevalentes em todas as amostras analisadas. As concentrações totais dos DDTs não excederam os padrões de qualidade exigidos $(\leq 3,89 \mathrm{ng} / \mathrm{g}-1)$, indicando que estes não representavam risco ecológico na área de estudo, semelhante aos estudos de Cui; Wei; Wang, 2016 e Liu e colaboradores., 2016 que também encontraram concentrações de DDTs abaixo do nível de risco previsto. Em contrapartida, $54 \%$ do total da concentração de $\mathrm{HCHs}$ excedeu o padrão exigido $\left(\leq 0,32 \mathrm{ng} / \mathrm{g}^{-1}\right)$, sugerindo que estes sim poderiam representar riscos ecológicos na região estudada.

Outro estudo também realizado na China por $\mathrm{Wu}$ e colaboradores., (2014), realizou a detecção de PCOs em amostras de águas localizadas entre o rio Yangtze e o lago Taihu, relatando que os DDTs, HCHs, aldrinas e heptacloros apresentaram as maiores concentrações médias, que foram de 52,46, 43,71, 40,95 e $34,29 \mathrm{ng} / \mathrm{L}^{-1}$ respectivamente. Os autores indicaram que o $\alpha-\mathrm{HCH}$ $\left(1,85 \times 10^{-6}\right)$, heptacloro $\left(4,97 \times 10^{-6}\right)$, heptacloro epóxido $\left(5,62 \times 10^{-6}\right)$, aldrina $\left(23,55 \times 10^{-6}\right)$ e dieldrina $\left(10,74 \times 10^{-6}\right)$ foram os que apresentaram maiores níveis de risco à saúde humana, já que excederam o nível de risco aceitável $\left(\leq 1 \times 10^{-6}\right)$, enquanto que os níveis de p,p'- DDT $\left(0,015 \times 10^{-6}\right)$ e p,p-DDD $\left(0,08 \times 10^{-6}\right)$ não ofereciam risco algum.

Em East Lake, maior lago urbano na China, um estudo buscou determinar a concentração de diversos PCOs. Os achados demonstraram que as maiores concentrações encontradas foram de HCHs $(7,40 \mathrm{ng} / \mathrm{L})$, DDTs $(5,70 \mathrm{ng} / \mathrm{L})$ e heptacloro $(2,71 \mathrm{ng} / \mathrm{L})$. Observou-se que os DDTs e HCHs poderiam trazer riscos a saúde humana no caso de ingestão da água, visto que os seus valores máximos excederam o limiar de risco aceitável $\left(\leq 1 \times 10^{-7}\right)$, que foram de 2,90 x 10-7 para os DDTs e 1,43 x 10-7 para os HCHs, sendo este último achado semelhante ao estudo de Wu e colaboradores., (2014) que também relacionaram o risco à saúde humana com os níveis de HCHs. Já em relação ao risco ecotoxicológico, observou-se que o nível de risco estabelecido $(\leq 1 \mathrm{x}$ 
$\left.10^{-5}\right)$ foi excedido pelos p, $\mathrm{p}^{\prime}$-DDT $\left(5,87 \times 10^{-8}\right), \gamma$ - $\mathrm{HCH}\left(8,24 \times 10^{-8}\right)$ e heptacloro $\left(1,86 \times 10^{-5}\right)$, concluindo que esses poderiam desencadear efeitos adversos nas formas de vida aquática (YANG et al., 2013).

Ogbeide; Tongo; Ezemonye, 2016, realizaram realizaram estudo com o objetivo de avaliar a concentração de PCOs em amostras de sedimentos em três regiões (Illushi, Ogbesse e Owan) com intensas atividades agrícolas na Nigéria. Os resultados demonstraram que a concentração média dos $\mathrm{HCHs}$ $(4,62 \mu \mathrm{g} / \mathrm{g} / \mathrm{dw})$ nas três regiões foram maiores em comparação com o DDT $(1,02 \mu \mathrm{g} / \mathrm{g} / \mathrm{dw})$, concluindo que as águas das três regiões não ofereciam risco ecotoxicológico, com concentrações abaixo dos padrões estabelecidos ( $\leq 6 \mu \mathrm{g} / \mathrm{g} / \mathrm{dw}$ para os HCHs e $\leq 4,2 \mu \mathrm{g} / \mathrm{g} / \mathrm{dw}$ para os DDTs). Esses achados foram similares aos de dois estudos feitos na China (CUI; WEI; WANG, 2016; LIU et al., 2016) que também encontraram concentrações de HCHs e DDTs que não ofereciam risco ecotoxicológico.

Um estudo realizado com águas de dois rios (Wedecha e Belbela) e três lagoas de um sistema de irrigação situado na região de Oramia, Etiópia, teve o objetivo de rastrear o nível de PCOs nessa área. O g-clordano $(10,1 \mu \mathrm{g} / \mathrm{L})$, b-HCB $(2,72 \mu \mathrm{g} / \mathrm{L})$, a-clordano $(0,192 \mu \mathrm{g} / \mathrm{L})$ e heptacloro epóxido B $(0,115 \mu \mathrm{g} / \mathrm{L})$ apresentaram maiores concentrações, excedendo os níveis padrões para águas superficiais exigidos pela legislação na qual os autores se basearam $(0,1 \mu \mathrm{g} / \mathrm{L}$ para g-clordano, b-HCB e a-clordano e $0,03 \mu \mathrm{g} / \mathrm{L}$ para o heptacloro epóxido B), concluindo que estes PCOs poderiam apresentar alto risco à saúde humana no caso de ingestão da água das regiões analisadas (TEKLU; ADRIAANSE; BRINK, 2016).

Além da detecção de PCOs na água para avaliação do risco ecotoxicológico, os estudos que fazem a detecção desses poluentes em humanos também são de grande importância, pois conseguem associar a exposição humana a partir dos níveis de PCOs encontrados com o desenvolvimento de doenças relacionadas ao metabolismo de algumas biomoléculas, dentre elas a DM2, que por ser associada a vários fatores de risco, também pode se relacionar com a exposição aos PCOs.

Diversos relatos na literatura científica têm demonstrado associações positivas entre as concentrações de PCOs com o risco de desenvolvimento de DM2, sendo que diversos fatores de risco com IMC, idade, níveis de colesterol e triglicerídeos também são investigados e levados em conta nesses estudos, com o objetivo de aumentar a significância dessas associações. Dentre os PCOs mais encontrados e associados positivamente com o risco de DM2 nesses estudos, são mais prevalentes os DDTs, HCB e HCHs.

Um estudo realizado com 280 adultos sauditas, sendo 136 (49\%) com diagnóstico de DM2, buscou associar as concentrações de DDTs com o risco de DM2. Observou-se que os pacientes diabéticos do estudo possuíam as maiores concentrações médias de DDTs $(18,3 \mathrm{ng} / \mathrm{mL})$ quando comparados aos não diabéticos $(11,8 \mathrm{ng} / \mathrm{mL})$. Nos pacientes diabéticos também foram 
relatadas maiores concentrações de colesterol, triglicerídeos e insulina, além de apresentarem as médias de idade e peso mais elevadas, mostrando assim forte associação entre a alta concentração de DDTs com esses outros fatores de risco nos pacientes com DM2 (AL-OTHMAN; ABD-ALRAHMAN; AL-DAGHRI, 2014).

Os achados de Al-Othman; Abd-Alrahman; Al-Daghri, 2014 foram consistentes com os de um estudo feito no Sul da Espanha acerca da associação da carga corporal de PCOs com o risco de DM2, onde Arrebola e colaboradores., (2013) encontraram maiores concentrações de DDTs nos 34 (8,8\%) pacientes que foram diagnosticados com DM2. A associação da concentração de DDTs com DM2 também foi positiva nos pacientes diabéticos que tinham idade e IMC mais elevados, indicando que as altas concentrações de DDTs podem estar relacionadas com pacientes que apresentam esses dois fatores de risco.

Ainda de acordo com os dois estudos anteriores, Kim e colaboradores., (2014) em um estudo com pacientes que tinham patologias pré-existentes relacionadas ao fígado, também encontraram os DDTs em maiores concentrações nos pacientes diabéticos com concentrações de triglicerídeos e idade elevados, e mostraram associação positiva com a DM2. No entanto, os autores enfatizam que essa associação pode não ser verdadeira em alguns casos, visto que alguns pacientes desse estudo tinham lesões hepáticas, e, nessa situação, pode haver alteração do metabolismo de lipídios e de glicose, levando a descompensação de seus valores.

Os achados de Lee e colaboradores., (2017) também se associam positivamente com os estudos anteriores. Os autores realizaram um estudo com 200 participantes de um hospital universitário, com o objetivo de determinar as concentrações séricas de PCOs e associar com o funcionamento das células $\beta$-pancreáticas a partir da dosagem de glicose e os valores de insulina e peptídeo c. Os resultados obtidos demonstraram que os pacientes que apresentaram altas concentrações, principalmente de DDTs, tiveram os valores de glicose e insulina maiores, indicando a sensibilidade a insulina reduzida, concluindo que a exposição crônica a esses PCOs pode induzir o mal funcionamento das células $\beta$-pancreáticas, através de mecanismos que ainda devem ser melhor elucidados.

Um estudo realizado com 300 indivíduos (149 com diagnóstico de DM2) que residiam próximos a áreas que passavam por frequentes aplicações de pesticidas, teve o objetivo de associar essa exposição com o risco de DM2. Os achados mostraram uma associação positiva com o risco de DM2 apenas com um dos metabólitos de DDT, o $o, p^{\prime}$ - DDT, quando comparado e ajustado com idade elevada, enquanto que o HCB foi associado negativamente com o risco de DM2, quando também comparado a esse fator (GRICE et al., 2017).

Aminov e colaboradores., (2016) realizaram um estudo sobre a prevalência de DM2 relacionada a concentrações plasmáticas de PCOs, onde 111 dos 601 participantes tinham diagnóstico de DM2. Os PCOs detectados no 
estudo foram o DDE (metabólito do DDT), Mirex e HCB. Apesar do DDE ser o encontrado em maiores concentrações médias na população total do estudo, os autores relataram que o HCB era o que podia estar mais associado positivamente com a prevalência de DM2, pois quando ajustado e comparado com outros fatores como idade e IMC elevados e altas concentrações de lipídios, foi o que apresentou maior associação positiva, sendo esse achado diferente do relatado no estudo de Grice e colaboradores., (2017), que associaram negativamente o $\mathrm{HCB}$ com o risco de DM2.

Em um estudo feito com caucasianos e afro americanos, sendo 114 deles com diagnóstico de DM2, foi relatado que 193 indivíduos tiveram níveis detectáveis de DDE, 49 de trans-nonacloro e 80 de oxyclordano. O DDE foi o encontrado em maiores concentrações médias, principalmente nos pacientes com DM2 (317,5 ng/g de lipídio). A associação com DM2 foi positiva para o DDE quando comparado e ajustado com os outros fatores investigados, como concentração de triglicerídeos, idade e IMC e glicose, que foram todos mais elevados nos pacientes diabéticos que tiveram níveis detectáveis de DDE, sendo esse achado divergente do estudo de Aminov e colaboradores., 2016, que mesmo encontrando o DDE em maiores concentrações médias, não o associaram positivamente ao risco de DM2 quando comparados com idade e IMC elevados (EDEN et al., 2014).

Al-Othman e colaboradores., (2014) investigaram HCHs em uma população de 280 sauditas, sendo 136 (49\%) com diagnóstico de DM2. Os HCHs foram mais prevalentes nos diabéticos, com uma concentração sérica média de $7,3 \mathrm{ng} / \mathrm{mL}$ nessa população. Observou-se também que os pacientes diabéticos apresentavam maiores níveis de glicose plasmática, insulina, triglicerídeos e IMC elevado, indicando que a exposição a esses $\mathrm{HCH}$ associada a esses fatores pode ser um fator desencadeante para o risco de DM2, enfatizando a necessidade de investigação de outros fatores de risco, para aumentar a significância dessas associações.

\section{CONCLUSÃO}

Diante dos dados da literatura científica presentes nesse estudo, conclui-se que a contaminação da água por PCOs pode oferecer grave risco ecotoxicológico a saúde humana, já que os estudos relataram que muitos PCOs como os DDTs, HCHs, heptacloros, clordanos e aldrinas podem estar presentes em altas concentrações na água por tempo prolongado, pois seu lento processo de degradação natural aumenta a sua persistência no meio ambiente. Através dos estudos, foi possível observar que muitos dos PCOs encontrados vinham de aplicações históricas, já que muitos deles foram banidos em diversos países há muitos anos em função da alta toxicidade. No entanto, alguns estudos indicavam o uso recente de alguns PCOs, sendo esse achado importante para alertar sobre a responsabilidade social acerca do meio ambiente, enfatizando 
a criação de estudos e programas de monitoramento ambiental que controlem com urgência e eficácia o uso desses PCOs.

Os achados dessa revisão também demonstraram as diversas associações positivas que podem ocorrer entre a exposição humana a PCOs como DDTs, $\mathrm{HCH}$ e $\mathrm{HCB}$ com o desenvolvimento de DM2, principalmente em pacientes que apresentam outros fatores de risco relacionados à DM2, como IMC, concentração de triglicerídeos e idade elevados. Por fim, sugere-se a importância da criação de estudos futuros que busquem entender a fisiopatologia da DM2 associada aos PCOs, elucidando os mecanismos biológicos pelos quais os PCOs interagem com as células $\beta$-pancreáticas, afetando a secreção de insulina e influenciando na homeostase da glicose.

\section{REFERÊNCIAS}

AL-OTHMAN, AbdulAziz A.; ABD-ALRAHMAN, Sherif H.; AL-DAGHRI, Nasser M. DDT and its metabolites are linked to increased risk of type 2 diabetes among Saudi adults: a cross-sectional study. Environmental Science and Pollution Research, [s.l], v. 22, n. 1, p. 379-386, jul. 2014.

AL-OTHMAN, Abdulaziz et al. Strong associations between the pesticide hexachlorocyclohexane and type 2 diabetes in Saudi adults. International Journal of Environmental Research and Public Health, [s.1], v. 11, n. 9, p. 8984-8995, ago. 2014.

AMINOV, Zafar et al. Diabetes prevalence in relation to serum concentrations of polychlorinated biphenyl (PCB) Congener groups and three chlorinated pesticides in a native american population. Environmental Health Perspectives, [s.l], v. 124, n. 9, p. 1376, set. 2016.

ARREBOLA, Juan P. et al. Adipose tissue concentrations of persistent organic pollutants and prevalence of type 2 diabetes in adults from Southern Spain.

Environmental Research, [s.l], v. 122, s/n, p. 31-37, abr. 2013.

BAHIA, Camila Alves; GUIMARÃES, Raphael Mendonça; ASMUS, Carmen Ildes Rodrigues Fróes. Alterações nos marcadores hepáticos decorrentes da exposição ambiental a organoclorados no Brasil. Cadernos Saúde Coletiva, Rio de Janeiro, v. 22, n. 2, p. 133-141. 2014.

CUI, Lili; WEI, Liangfu; WANG, Jun. Residues of organochlorine pesticides in surface water of a megacity in central China: seasonal-spatial distribution and fate in Wuhan. Environmental Science and Pollution Research, [s.1], v. 24, n. 2, p. 1975-1986, out. 2016.

DEL GRANDE, Marcelo; REZENDE, Maria Olímpia Oliveira; ROCHA, Odete.

Distribuição de compostos organoclorados nas águas e sedimentos da bacia do Rio Piracicaba/SP-Brasil. Química Nova, [s.1], v. 26, n. 5, p. 678-686. 2003. 
EDEN, Paul R. et al. Association of type 2 diabetes mellitus with plasma organochlorine compound concentrations. Journal of Exposure Science and Environmental Epidemiology, [s.1], v. 26, n. 2, p. 207-213, out. 2014.

FLORES, Araceli Verônica et al. Organoclorados: um problema de saúde pública. Ambiente \& Sociedade, [s.1], v. 7, n. 2, p. 111-125, jul./dez. 2004.

GAO, Li R. et al. Concentrations and distributions of 18 organochlorine pesticides listed in the Stockholm Convention in surface sediments from the Liaohe River basin, China. Journal of Environmental Science and Health, Part B, [s.1], v. 50, n. 5, p. 322330, out. 2015.

GRICE, Brian A. et al. Associations between persistent organic pollutants, type 2 diabetes, diabetic nephropathy and mortality. Occupational and Environmental Medicine, [s.1], v. 74, s/n, p. 521-527, mar. 2017.

HUANG, Yumei et al. Organochlorine pesticides in the atmosphere and surface water from the equatorial Indian Ocean: enantiomeric signatures, sources, and fate. Environmental Science \& Technology, [s.1], v. 47, n. 23, p. 13395-13403, nov. 2013.

JAYARAJ, Ravindran; MEGHA, Pankajshan; SREEDEV, Puthur. Organochlorine pesticides, their toxic effects on living organisms and their fate in the environment. Interdisciplinary Toxicology, [s.1], v. 9, n. 3-4, p. 90-100. 2016.

JUNTARAWIJIT, Chudchawal; JUNTARAWIJIT, Yuwayong. Association between diabetes and pesticides: a case-control study among Thai farmers. Environmental Health and Preventive Medicine, [s.1], v. 23, n. 3, p. 1-10, jan. 2018.

KIM, Ki-Su et al. Associations of organochlorine pesticides and polychlorinated biphenyls in visceral vs. subcutaneous adipose tissue with type 2 diabetes and insulin resistance. Chemosphere, [s.1], v. 94, s/n, p. 151-157, jan. 2014.

LEE, Yu-Mi et al. Persistent Organic Pollutants Impair Insulin Secretory Function of Pancreatic Beta-Cells: Human and in Vitro Evidence. Diabetes, [s.1], v. 66, n. 10, p. 2669-2680, jul. 2017.

LIU, Jia et al. Contamination characteristics of organochlorine pesticides in multimatrix sampling of the Hanjiang River Basin, southeast China. Chemosphere, [s.1], v. 163, s/n, p. 35-43, nov. 2016.

MOSTAFALOU, Sara; ABDOLLAHI, Mohammad. Pesticides: an update of human exposure and toxicity. Archives of Toxicology, [s.1], v. 91, n. 2, p. 549-599, out. 2016.

OGBEIDE, Ozekeke; TONGO, Isioma; EZEMONYE, Lawrence. Assessing the distribution and human health risk of organochlorine pesticide residues in sediments from selected rivers. Chemosphere, [s.1], v. 144, s/n, p. 1319-1326, fev. 2016.

PRATES, Crislaine Batista; GEBARA, Sâmya Soler; POPPI, Nilva Ré. Análise de pesticidas organoclorados em água usando a microextração em fase sólida por 
headspace com cromatografia gasosa e espectrometria de massas. Química Nova, [s.1], v.34, n. 7, p. 1260-1264, abr. 2011.

RISSATO, Sandra Regina et al. Determinação de pesticidas organoclorados em água de manancial, água potável e solo na região de Bauru (SP). Química Nova, [s.1], v. 27, n. 5, p. 739-743, jun. 2004.

TANG, Mengling et al. Exposure to organochlorine pollutants and type 2 diabetes: a systematic review and meta-analysis. PloS One, [s.1], v. 9, n. 10, p. 1-12, out. 2014.

TEKLU, Berhan M.; ADRIAANSE, Paulien I.; VAN DEN BRINK, Paul J. Monitoring and risk assessment of pesticides in irrigation systems in Debra Zeit, Ethiopia.

Chemosphere, [s.1], v. 161, s/n, p. 280-291, out. 2016.

VARGAS-GONZÁLEZ, Héctor H. et al. Persistent organic pollutants (POPs) in populations of the clam Chione californiensis in coastal lagoons of the Gulf of California. Journal of Environmental Science and Health, Part B, [s.1], v. 51, n. 7, p. 435-445, abr. 2016.

WU, Chunfa et al. Concentrations and potential health hazards of organochlorine pesticides in shallow groundwater of Taihu Lake region, China. Science of the Total Environment, [s.1], v. 470, s/n, p. 1047-1055, fev. 2014.

YANG, Yuyi et al. Concentrations, distributions, sources, and risk assessment of organochlorine pesticides in surface water of the East Lake, China. Environmental Science and Pollution Research, [s.1], v. 21, n. 4, p. 3041-3050, nov. 2013.

ZHOU, Rongbing et al. Distribution of organochlorine pesticides in surface water and sediments from Qiantang River, East China. Journal of Hazardous Materials, [s.l], v. 137, n. 1, p. 68-75, mar. 2006. 